\title{
Patellar Tilt in Two Modern Total Knee Arthroplasty Systems: A Matched-Cohort Analysis
}

\author{
Braeden W. Estes BS, ${ }^{1}$ Lauren Pitz BS, ${ }^{1}$ \\ Evan R. Deckard BSE, ${ }^{2}$ R. Michael Meneghini MD ${ }^{2}$ \\ ${ }^{1}$ Indiana University School of Medicine \\ 2 Indiana University School of Medicine, Department of Orthopaedic Surgery
}

Background and Hypothesis: Excessive patellar tilt is undesirable in total knee arthroplasty (TKA) and can result in patellofemoral complications and premature failure/wear. Few studies have investigated patellar tilt between TKA systems with different femoral component geometries and bearing articulations. The purpose of this study was to (1) compare patellar tilt between TKA systems with differing femoral component geometries and (2) evaluate related differences in patient-reported outcome measures (PROMS). The study hypothesis was neutral patellar tilt would correlate with improved postoperative PROMS.

Experimental Design or Project Methods: 380 symmetric trochlear groove TKAs were matched to 380 asymmetric lateralized trochlear groove TKAs on age, sex, body mass index, and preoperative patellar tilt. All cases were performed by one surgeon between $12 / 2010$ and 10/2018. Patellar tilt (in degrees) was measured by two independent blinded raters on preoperative and 4-week postoperative merchant view radiographs per the modern Knee Society Radiographic Evaluation System. Prospectively collected and validated PROMS including UCLA Activity Level, components of the Knee Society Score, and Likert satisfaction were evaluated at minimum one-year.

Results: There were no differences between study cohorts for demographics, covariates, or preoperative patellar tilt $(p \geq 0.479)$. Asymmetric lateralized trochlear groove TKAs had significantly less postoperative patellar tilt compared with symmetric trochlear groove TKAs $(p<0.001)$. Patellar tilt and femoral implant type had no correlation to pain scores (Knee Society), UCLA Activity Level, or satisfaction ( $p \geq 0.138$ ); however, postoperative patellar tilt was significantly less for patients who stated their knee "always" felt normal compared to patients who stated their knee "sometimes" felt normal but not "never" felt normal $(p=0.033)$.

Conclusion and Potential Impact: Findings suggest implant type and patellar tilt have minimal effect on overall PROMS. However, minimizing patella maltracking may provide patients with a more normal feeling TKA and could potentially provide long-term benefits of preventing polyethylene wear or other complications leading to premature implant failure. 